\title{
Use of rumination and activity monitoring for the identification of dairy cows with health disorders: Part I. Metabolic and digestive disorders
}

\author{
M. L. Stangaferro, R. Wijma, L. S. Caixeta, ${ }^{1}$ M. A. Al-Abri, ${ }^{2}$ and J. O. Giordano ${ }^{3}$ \\ Department of Animal Science, Cornell University, Ithaca, NY 14853
}

\section{ABSTRACT}

The objectives of this study were to evaluate (1) the performance of an automated health-monitoring system (AHMS) to identify cows with metabolic and digestive disorders - including displaced abomasum, ketosis, and indigestion-based on an alert system (health index score, HIS) that combines rumination time and physical activity; (2) the number of days between the first HIS alert and clinical diagnosis (CD) of the disorders by farm personnel; and (3) the daily rumination time, physical activity, and HIS patterns around CD. Holstein cattle ( $\mathrm{n}=1,121 ; 451$ nulliparous and 670 multiparous) were fitted with a neck-mounted electronic rumination and activity monitoring tag (HR Tags, SCR Dairy, Netanya, Israel) from at least -21 to $80 \mathrm{~d}$ in milk (DIM). Raw data collected in 2-h periods were summarized per $24 \mathrm{~h}$ as daily rumination and activity. A HIS (0 to 100 arbitrary units) was calculated daily for individual cows with an algorithm that used rumination and activity. A positive HIS outcome was defined as a HIS of $<86$ during at least $1 \mathrm{~d}$ from -5 to $2 \mathrm{~d}$ after $\mathrm{CD}$. Blood concentrations of nonesterified fatty acids, $\beta$-hydroxybutyrate, total calcium, and haptoglobin were determined in a subgroup of cows ( $\mathrm{n}=$ $459)$ at $-11 \pm 3,-4 \pm 3,0,3 \pm 1,7 \pm 1,14 \pm 1$, and $28 \pm 1$ DIM. The sensitivity of the HIS was $98 \%$ [95\% confidence interval (CI): 93, 100] for displaced abomasum $(\mathrm{n}=41) ; 91 \%(95 \%$ CI: 83, 99) for ketosis $(\mathrm{n}=$ $54) ; 89 \%$ (95\% CI: 68,100$)$ for indigestion $(\mathrm{n}=9)$; and 93\% (95\% CI: 89, 98) for all metabolic and digestive disorders combined $(\mathrm{n}=104)$. Days (mean and $95 \%$ CI) from the first positive HIS $<86$ and CD were -3

\footnotetext{
Received January 18, 2016.

Accepted May 21, 2016.

${ }^{1}$ Current address: Department of Clinical Sciences, College of Veterinary Medicine and Biomedical Sciences, Colorado State University, Fort Collins, CO 80523.

${ }^{2}$ Current address: Department of Animal and Veterinary Sciences, College of Agriculture and Marine Sciences, Sultan Qaboos University, Muscat, Sultanate of Oman.

${ }^{3}$ Corresponding author: jog25@cornell.edu
}

$(-3.7,-2.3),-1.6(-2.3,-1.0),-0.5(-1.5,0.5)$, and $-2.1(-2.5,-1.6)$ for displaced abomasum, ketosis, indigestion, and all metabolic and digestive disorders, respectively. The patterns of rumination, activity, and HIS for cows flagged by the AHMS were characterized by lower levels than for cows without a health disorder and cows not flagged by the AHMS from -5 to $5 \mathrm{~d}$ after $\mathrm{CD}$, depending on the disorder and parameter. Differences between cows without health disorders and those flagged by the AHMS for blood markers of metabolic and health status confirmed the observations of the CD and AHMS alerts. The overall sensitivity and timing of the AHMS alerts for cows with metabolic and digestive disorders indicated that AHMS that combine rumination and activity could be a useful tool for identifying cows with metabolic and digestive disorders.

Key words: rumination, activity, metabolic disorder, digestive disorder

\section{INTRODUCTION}

Health disorders in the early postpartum period affect a substantial proportion of lactating dairy cows, with negative results for their health, welfare, and performance (Ingvartsen, 2006). Metabolic and digestive disorders such as ketosis, displaced abomasum (DA), and indigestion are detrimental to cow well-being and farm profitability because they cause losses in milk production (Gröhn et al., 1998; Bareille et al., 2003; Edwards and Tozer, 2004), increase the risk of culling and death (Gröhn et al., 1998; Pinedo et al., 2010; Seifi et al., 2011), increase treatment costs (Kaneene and Hurd, 1990; Bartlett et al., 1995), and impair reproductive performance (Raizman and Santos, 2002; Ribeiro et al., 2013; Vercouteren et al., 2015).

For dairy operations, the burden of metabolic and digestive diseases is exacerbated by the additional effort and costs associated with implementing the monitoring necessary to identify cows with these disorders (McArt et al., 2015). Although the intensity of health-monitoring programs varies widely among farms, protocols that include a systematic evaluation of cow health status once or twice per day within the 
first 1 to 3 wk after calving are common (Risco and Melendez Ratamal, 2011; Espadamala et al., 2015). Such protocols may include evaluation of attitude, appetite, locomotion, and rectal temperature, as well as auscultation, palpation, and collection of bodily fluids for cow-side or laboratory testing (LeBlanc, 2010; Risco and Melendez Ratamal, 2011). Performing these evaluations and diagnostic tests for a large number of cows can be time-consuming and labor-intensive. Moreover, cow behavior and time budgets are disrupted, because in free-stall herds, cows are examined while restrained in self-locking head gates or in palpation rails after they have been sorted from their herd mates. In this regard, automated monitoring of cow behavior and physiological parameters using non-invasive sensors may help reduce the burden of health-monitoring programs. Sensor-generated data could be used alone or with traditional health-monitoring protocols to identify cows with health disorders (Rutten et al., 2013; Lukas et al., 2015). Furthermore, continuous monitoring of behavior and physiological parameters may allow for the detection of subtle changes before evident clinical signs appear. Earlier disease detection may benefit cows by preventing progression and improving response to treatment.

In recent years, multiple devices have been developed and implemented by the dairy industry to automatically monitor behavior and physiological parameters (Rutten et al., 2013; Ferrero et al., 2014; Barkema et al., 2015). Physical activity levels and rumination time are 2 parameters that are currently available for monitoring cow health. Cows with health disorders would be expected to demonstrate alterations in their activity and rumination patterns of sufficient magnitude to be detected by specific algorithms or visual inspection of data. Indeed, previous studies have found that rumination time and activity were associated with clinical and subclinical health disorders (Soriani et al., 2012; Gaspardy et al., 2014; Liboreiro et al., 2015). These studies have documented general trends and changes in rumination and activity patterns for some disorders, but the performance of automated health-monitoring systems (AHMS) that use rumination and activity to detect cows with metabolic and digestive disorders has not been well documented. In addition, more information is needed about the patterns of rumination and activity around the timing of clinical diagnosis (CD) of metabolic and digestive disorders in dairy cows.

We hypothesized that an AHMS that continuously monitors rumination and activity would be able to identify cows with metabolic and digestive disorders. Also, we expected that changes in rumination and activity before evident clinical signs of disease would result in earlier identification of disease. The objectives of this study were to evaluate (1) the performance of an automated rumination and physical activity monitoring system to identify cows with metabolic and digestive disorders; (2) the interval between the AHMS alert based on a health index score (HIS) and the day of CD by farm personnel; and (3) the rumination, activity, and AHMS-generated alert pattern for cows with the disorders of interest. We also used markers of energy status [nonesterified fatty acids (NEFA) and BHB], mineral status (total plasma Ca), and systemic inflammation (haptoglobin) to complement the diagnosis of health disorders and the performance of the AHMS alert.

\section{MATERIALS AND METHODS}

\section{Animals and Management}

All procedures were approved by the Institutional Animal Care and Use Committee of Cornell University. This study was conducted from November 2013 to October 2014 at a commercial dairy operation in Cayuga County, New York State. Holstein cows ( $\mathrm{n}=1,121$; 451 nulliparous and 670 multiparous) were enrolled in the study at approximately 240 to $250 \mathrm{~d}$ of gestation. During the prepartum period, cows were grouped by parity (nulliparous vs. parous) and housed in a freestall barn with pens that had 3 rows of stalls. Cows were monitored for signs of calving every $45 \mathrm{~min}$ by farm personnel. At the first signs of calving (visualization of the allantoic sac through the vulva, restlessness, discomfort), cows were moved to a loose housing pen to evaluate and assist calving, or both. Immediately after calving, cows were moved to another loose housing pen with straw bedding for $1 \mathrm{~d}$. Thereafter, cows were moved to a postpartum pen if farm personnel considered them healthy. Primiparous and multiparous cows were commingled in the postpartum pen for about the first 30 DIM. Thereafter, cows were moved to pens based on lactation number (first, second, third and fourth or more) for the rest of their lactation. Cows with health disorders that were treated with antibiotics and required milk withdrawal were placed in a separate pen, and their milk was discarded until it was saleable.

Cows were milked 3 times per day, and individual milk yield and conductivity were recorded at each milking (Afimilk, Kibbutz Afikim, Israel). The projected 305-d milk production for cows that calved during the study period was $13,036 \mathrm{~kg}$. Cows had ad libitum access to feed and water and were fed a TMR once daily. A detailed description of the diets fed during the study is presented in Table 1. 


\section{Study Design}

The study followed an observational prospective cohort design. Cows were fitted with a neck-mounted electronic rumination and activity monitoring tag (HR Tags; SCR Dairy, Netanya, Israel) approximately $4 \mathrm{wk}$ before calving to monitor rumination time and physical activity from at least $21 \mathrm{~d}$ before expected calving until at least $80 \mathrm{~d}$ after calving (Figure 1). Rumination raw data were recorded in minutes per $2 \mathrm{~h}$ interval; activity raw data were also recorded every $2 \mathrm{~h}$ as an arbitrary number. Activity level was determined by a 3 -axis accelerometer that recorded the speed and angle of head movements. Based on these 2 parameters, the system software generated an arbitrary number from 0 to 255 . These data were summarized as an arbitrary number per unit of time $(2 \mathrm{~h}$ or $24 \mathrm{~h})$. Data from individual tags were transferred to the system software (Dataflow; SCR Dairy) automatically every $20 \mathrm{~min}$ via antennas in the barns. Based on rumination and activity data, the system software generated a HIS (0 to 100 arbitrary units) for each cow using a series of internal algorithms (proprietary to SCR Dairy). A HIS of 100 arbitrary units represents a cow with an ideal pattern of rumination and activity; a HIS value of $<86$ arbitrary units may be indicative of a health disorder. Thus, HIS is designed to serve as an alert system to help dairy farm personnel identify cows for further clinical examination. A HIS report was generated daily to include cows with $<86$ arbitrary units (as determined by SCR) and stored for evaluation by the research group. During the study, farm personnel did not have access to the HIS report or any information generated by the AHMS.

\section{Clinical Examination and Case Definitions}

Before initiation of the study, the health-monitoring program was defined and discussed by the research team, the herd veterinarian, and the farm personnel responsible for conducting daily health monitoring. All

Table 1. Summary of formulated feed ingredient amounts and formulated nutrient composition of the diets during the study

\begin{tabular}{|c|c|c|c|c|}
\hline \multirow[b]{2}{*}{ Feed ingredient or nutrient } & \multicolumn{2}{|c|}{ Prepartum } & \multicolumn{2}{|c|}{ Postpartum } \\
\hline & Heifers & Cows & $\begin{array}{c}\text { Fresh } \\
(<30 \text { DIM })\end{array}$ & $\begin{array}{c}\text { High } \\
(>30 \text { DIM })\end{array}$ \\
\hline \multicolumn{5}{|l|}{ Ingredient $(\%$ of $\mathrm{DM})$} \\
\hline Conventional corn silage & 46.6 & 46.2 & 23.2 & 25.5 \\
\hline Brown midrib corn silage & - & - & 16.0 & 12.5 \\
\hline Alfalfa silage & - & - & 10.1 & 13.6 \\
\hline Grass hay & 14.5 & 14.2 & - & - \\
\hline Wheat straw & 14.5 & 14.2 & 3.9 & - \\
\hline Corn grain finely ground & - & - & 17.5 & 16.7 \\
\hline Citrus pulp (dry) & - & - & - & 4.1 \\
\hline Soy Plus ${ }^{1}$ & - & - & 6.0 & 5.2 \\
\hline Canola meal (solvent) & - & - & 4.0 & 5.2 \\
\hline Molasses & - & - & 5.1 & 4.3 \\
\hline Acid whey (liquid) & 2.2 & 2.1 & 0.9 & 0.7 \\
\hline Prefresh concentrate mix & 22.1 & 21.9 & - & - \\
\hline Fresh concentrate mix & - & - & 13.4 & - \\
\hline High concentrate mix & - & - & - & 12.2 \\
\hline Anionic mineral supplement & - & 1.3 & - & - \\
\hline \multicolumn{5}{|l|}{ Nutrient composition (DM basis) } \\
\hline $\mathrm{CP}(\%)$ & 14.3 & 13.9 & 16.2 & 17.2 \\
\hline Ether extract (\%) & 3.8 & 3.2 & 5.6 & 5.3 \\
\hline $\mathrm{NDF}(\%)$ & 42.8 & 42.6 & 28.3 & 27.1 \\
\hline Starch $(\%)$ & 17.7 & 18.6 & 28.4 & 27.2 \\
\hline $\mathrm{NFC}^{2}(\%)$ & 30.2 & 31.2 & 42.7 & 43.1 \\
\hline Ash $(\%)$ & 9.5 & 9.5 & 7.3 & 7.6 \\
\hline Calcium (\%) & 1.77 & 1.75 & 0.72 & 0.79 \\
\hline Phosphorus (\%) & 0.35 & 0.36 & 0.40 & 0.40 \\
\hline Magnesium (\%) & 0.41 & 0.47 & 0.31 & 0.31 \\
\hline Sodium $(\%)$ & 0.08 & 0.06 & 0.38 & 0.47 \\
\hline Potassium (\%) & 1.27 & 1.27 & 1.48 & 1.48 \\
\hline Chlorine $(\%)$ & 0.44 & 0.59 & 0.64 & 0.56 \\
\hline Sulfur $(\%)$ & 0.43 & 0.48 & 0.24 & 0.24 \\
\hline $\mathrm{DCAD}^{3}(\mathrm{mEq} / \mathrm{kg})$ & -34 & -118 & 213 & 279 \\
\hline
\end{tabular}

${ }^{1}$ West Central, Ralston, IA.

${ }^{2}$ Calculated as $100-\mathrm{CP}$ - ether extract - NDF - ash.

${ }^{3}$ Calculated as $[(\mathrm{Na}+\mathrm{K})-(\mathrm{Cl}+\mathrm{S})]$. 


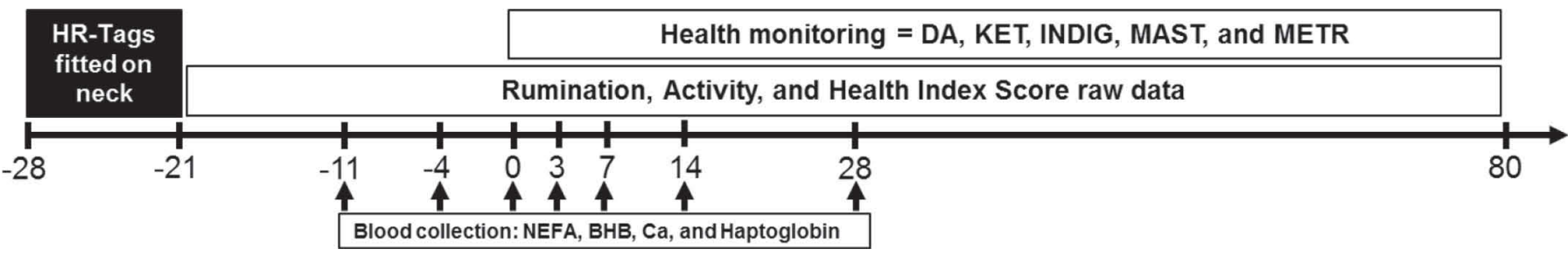

Figure 1. Graphical depiction of the study design. Cows were fitted with a neck-mounted electronic rumination and activity monitoring tag (HR Tags; SCR Dairy, Netanya, Israel) approximately $4 \mathrm{wk}$ before calving to monitor rumination and activity from at least $21 \mathrm{~d}$ before calving until at least 80 DIM. Rumination and activity raw data were recorded every $2 \mathrm{~h}$ and transferred to the automated health-monitoring system (AHMS) software. The AHMS uses an alert system to flag cows that may have a health disorder. The alert is based on a health index score (HIS; 0 to 100 arbitrary units) created daily for individual cows by combining rumination and activity data. The AHMS performance was evaluated using clinical diagnosis of health disorders by farm personnel as the reference test. The patterns of rumination, activity, and HIS were evaluated around the clinical diagnosis of the health disorders of interest. Health disorders monitored included displaced abomasum, ketosis, indigestion, mastitis, and metritis. Data for displaced abomasum, ketosis, and indigestion are presented in this manuscript; data for mastitis and metritis are presented in 2 companion manuscripts (Stangaferro et al., 2016a,b). Blood samples were collected at $-11 \pm 3,-4 \pm 3,0,3 \pm 1,7 \pm 1,14 \pm 1$, and $28 \pm 1 \mathrm{~d}$ relative to calving to determine circulating concentrations of nonesterified fatty acids (NEFA), BHB, total Ca, and haptoglobin. $\mathrm{DA}=$ displaced abomasum, KET $=$ ketosis, INDIG $=$ indigestion, MAST $=$ mastitis, and METR $=$ metritis.

farm personnel $(\mathrm{n}=5)$ conducted the clinical evaluation of cows on a daily basis ( 1 or 2 people per day) and all had 2 to $10 \mathrm{yr}$ of experience monitoring cow health at the time the study was conducted.

After calving, cows were examined daily from 1 to 10 DIM. The clinical examination included direct observation (general appearance and attitude, presence of fetal membranes outside the vulva, evaluation of vaginal discharge, foot health, udder health, and manure consistency), rectal temperature, urine ketones, and rumen auscultation. For any cow not diagnosed with metritis before 8 DIM, transrectal massage of the uterus was conducted to obtain and evaluate uterine discharge. Further, the progression of milk production and deviations in daily milk weights (difference between recorded and expected milk) were used as an aid in the identification of cows with health disorders during the entire lactation. Each disease of interest was defined, and case definitions were provided to farm personnel. Specific health disorders monitored included (1) retained placenta: failure to expel fetal membranes within $24 \mathrm{~h}$ after calving; (2) milk fever: fresh cow with low body temperature, muscle tremors, inability to rise, and in some cases lying down in abnormal reclining position with the head stretched over the back; (3) ketosis: decreased appetite, test at or above "moderate" using a urine ketone strip test (KetoStix, Bayer Diagnostics, Tarrytown, NY), and altered pattern of milk production (reduction from expected); (4) DA: movement of the fourth compartment of the stomach to an abnormal position on the right or left side of the abdomen, detected by auscultation of a "ping" sound with finger percussion; (5) indigestion: scant manure and lack of appetite with rumen and intestinal stasis; (6) metritis: watery, pink/brown, and fetid uterine discharge with or without fever (defined as rectal temperature $>39.5^{\circ} \mathrm{C}$ );
(7) clinical mastitis: swelling or pain in the udder, milk with an abnormal appearance (milk was stripped onto the floor and observed for flakes or clots), signs of udder inflammation possibly accompanied by depressed attitude, anorexia, and fever; (8) pneumonia: fever, increased respiratory rate and effort, nasal discharge, and malodorous breath; and (9) lameness: cows with locomotion score $\geq 3$ ( $1=$ normal locomotion and $5=$ severely lame).

After 10 DIM, cows were monitored daily following the same criteria, except that urine ketones were evaluated only in cows suspected of having ketosis, uterine discharge in cows suspected of having metritis, and rectal temperature in cows suspected of having metritis, mastitis, or other infectious disorders. Cows with health disorders after 10 DIM were monitored daily (or more frequently as needed) until recovery. Two consecutive episodes of the same disorder were evaluated separately when they occurred at least $7 \mathrm{~d}$ apart.

\section{Blood Collection and Laboratory Analyses}

Blood samples were collected on $\mathrm{d}-11 \pm 3$ and -4 \pm 3 prepartum, and $0,3 \pm 1,7 \pm 1,14 \pm 1$, and $28 \pm 1$ after calving (Figure 1) from a subgroup of cows $(\mathrm{n}=459)$. Blood was collected via puncture of the coccygeal vein or artery using evacuated tubes containing sodium heparin (Vacutainer; Becton Dickinson, Franklin Lakes, NJ). Samples were immediately placed on ice or refrigerated at $4^{\circ} \mathrm{C}$ and then transported to the laboratory for further processing (centrifugation at $2,000 \times g, 15 \mathrm{~min})$. Samples were stored at $-20^{\circ} \mathrm{C}$ until assayed. Plasma samples were analyzed on specific days relative to calving for NEFA (d -11, -4, 0, 3, 7, 14, 28), BHB (d 0, 3, 7, 14, 28), total Ca (d 0, 3, 7, 14), and haptoglobin $(\mathrm{d}-4,0,3,7,14,28)$. 
Plasma concentrations of NEFA were analyzed in triplicate by enzymatic analysis using a commercial kit (NEFA-HR2; Wako Diagnostic Inc., Richmond, VA). Plasma concentrations of BHB were analyzed in triplicate by enzymatic analysis (BHB dehydrogenase) following an adaptation of the Sigma kit 310-UV using enzyme 3-HBDH from Rhodopseudomonas sphaeroides (Roche Diagnostics Corp., Indianapolis, IN) and standards made from DL- $\beta$-hydroxybutyric acid sodium salt (Sigma-Aldrich, Saint Louis, MO). All spectrophotometric measurements were done using a Spectramax 190 microplate reader (Molecular Devices, Sunnyvale, CA). Intra- and interassay CV for the NEFA assay were 2.6 and $9.5 \%$, respectively, and for the BHB assay were 12.8 and $24.0 \%$, respectively.

Plasma total $\mathrm{Ca}$ concentrations were analyzed by spectrophotometric method using an enzymatic assay and following standard procedures for the Roche/ Hitachi Modular P Chemistry Analyzer (Roche Diagnostics, Rotkreuz, Switzerland) at the Animal Health Diagnostic Center of the Cornell University College of Veterinary Medicine (Ithaca, NY).

Haptoglobin concentrations were evaluated in duplicate using an enzymatic analysis that measures haptoglobin-hemoglobin complex by estimated differences in peroxidase activity as described in Bicalho et al. (2014). Estimations of haptoglobin concentrations were calculated against a standard curve from 0 to 2.5 $\mathrm{mg} / \mathrm{mL}$ (Molecular Innovations, Novi, MI). Samples with initial haptoglobin concentrations of $2.5 \mathrm{mg} / \mathrm{mL}$ were reanalyzed after dilution 1:4 in distilled water. Measurements were done using a Spectramax 190 microplate reader (Molecular Devices). Intra- and interassay CV for the haptoglobin assay were 10.5 and $20.6 \%$, respectively.

\section{Statistical Analysis}

System Performance. The main outcome of interest for this study was the ability of the HIS to correctly identify cows with a metabolic or digestive health disorder. Clinical diagnosis by farm personnel was used as the reference test. Because HIS does not confirm the occurrence of disease or indicate the type of disease, a positive outcome was defined as a HIS of $<86$ arbitrary units on at least $1 \mathrm{~d}$ during the $5 \mathrm{~d}$ before, the day of, or $2 \mathrm{~d}$ after the disease CD. The sensitivity and $95 \%$ CI of the HIS for each disease of interest (DA, ketosis, indigestion) was calculated using PROC FREQ in SAS (version 9.4; SAS Institute Inc., Cary, NC) and was defined as the ability of the HIS to correctly identify cows with a positive CD outcome. The overall sensitivity of HIS to identify all cows with metabolic and digestive disorders combined was also calculated. To evaluate the potential confounding effect of other health disorders (i.e., all disorders of interest, pneumonia, and lameness) on the sensitivity of HIS, we conducted 3 separate analyses. The first analysis included all cows diagnosed with DA, ketosis, or indigestion (regardless of the occurrence of another disorder during the range of interest around CD); a second analysis included cows diagnosed only with DA, ketosis, or indigestion during the range of interest around CD; and a third analysis included cows diagnosed with the disorder of interest and at least one other health disorder. Data for mastitis and metritis are presented in 2 companion manuscripts (Stangaferro et al., 2016a,b). We determined differences in the sensitivity of HIS between the subgroup of cows with only the disorder of interest and cows with the disorder of interest and at least another disorder by logistic regression using the events over trials option of PROC LOGISTIC in SAS. Retained placenta and milk fever were recorded, but these disorders were not included in the analysis because both are easily identified with a simple visual inspection of the cow. Pneumonia $(\mathrm{n}=31)$ and lameness $(\mathrm{n}=49)$ events were also recorded but were not included in the analysis because they were not of interest in the current study.

Interval Between the First Positive HIS Outcome and Clinical Diagnosis. To determine if HIS was capable of identifying cows with metabolic and digestive disorders earlier than CD by farm personnel, the interval (in days) between the first positive HIS outcome for each health event during the period of interest around CD ( $5 \mathrm{~d}$ before to $2 \mathrm{~d}$ after) and the day of $\mathrm{CD}$ was evaluated. For this analysis, which included only cows flagged by the AHMS, we compared the mean number of days from the first HIS positive outcome and the day of CD with a paired $t$-test conducted with the PROC TTEST in SAS.

Rumination, Activity, HIS, and Milk Production Relative to Clinical Diagnosis. Daily rumination time $(\mathrm{min} / \mathrm{d})$, daily activity (arbitrary units/d), and HIS (arbitrary units) were evaluated from $5 \mathrm{~d}$ before to $5 \mathrm{~d}$ after CD (d 0) for the first event of each disorder of interest. Before statistical analysis, we assessed the normality of the data for rumination, activity, and HIS using the Shapiro-Wilk statistic and graphical methods (histogram and Q-Q plot) and using PROC UNIVARIATE in SAS. Based on this analysis, no data transformations were necessary. For cows with each disorder of interest, cows were grouped according to the following criteria: health disorder diagnosed and HIS-positive (HI+; HIS $<86$ arbitrary units at least $1 \mathrm{~d}$ within $5 \mathrm{~d}$ before, the day of, and $2 \mathrm{~d}$ after $\mathrm{CD}$ ); health disorder diagnosed and HIS-negative (HI-; HIS $\geq 86$ arbitrary units from 5 d before, the day of, and 2 $\mathrm{d}$ after $\mathrm{CD}$ ); and $\mathrm{CD}$ negative (nondisease, $\mathbf{N D}$; cows 
not diagnosed with a health disorder during the study period). For cows in the ND group, we considered the average DIM at CD for cows with health disorders to be "day 0" (calculated for each disorder). Data were analyzed by ANOVA with repeated measurements using PROC MIXED in SAS. Models for each outcome of interest (rumination, activity, and HIS) included group (HI+, HI-, ND), time, group-by-time interaction, parity, and group-by-parity interaction as explanatory variables. The occurrence of another health disorder (i.e., all disorders of interest, pneumonia, and lameness) during the -5 to $5 \mathrm{~d}$ period after CD was offered as a categorical variable $(0=$ no occurrence and $1=$ occurrence) to the initial models to evaluate the potential effect of multiple disorders on the parameter of interest. The final model for each parameter of interest was selected by backward elimination of explanatory variables with $P>0.10$ and determination of the lowest value for the Akaike's information criterion. Cow within group was included as a random effect in all models. Cow was the subject of repeated measurements, and all models were run using an autoregressive (AR-1) covariance structure. Group, time, and group-by-time interaction were forced in all models. When the main effect or interaction between explanatory variables was significant, we used the LSD post hoc mean separation test to determine specific differences between groups of means.

Differences (in units of measurement) and percentage change between $5 \mathrm{~d}$ before $\mathrm{CD}$ and the day of the nadir for rumination, activity, HIS, and milk production for cows in the $\mathrm{HI}+, \mathrm{HI}-$, and ND groups were analyzed using PROC MIXED in SAS. The statistical models contained group (HI+, HI-, and ND), parity, and group-by-parity interaction as explanatory variables. The occurrence of another health disorder during the -5 to $5 \mathrm{~d}$ period after $\mathrm{CD}$ was also offered to the initial models. The final model for each variable of interest and the mean separation test were conducted as described.

Plasma Concentrations of NEFA, BHB, Calcium, and Haptoglobin. Depending on the parameter of interest, plasma samples were analyzed at different days relative to calving (NEFA: d $-11,-4,0,3,7,14$, and 28; BHB: d 0, 3, 7, 14, and 28; Ca: d 0, 3, 7, and 14; haptoglobin: $\mathrm{d}-4,0,3,7,14$, and 28). Cows were grouped in the same way as for evaluation of rumination, activity, and HIS pattern changes over time. Data were analyzed by ANOVA with repeated measurements using PROC MIXED in SAS as described for the other repeated measurement analyses.

Data for proportions are presented as arithmetical means and 95\% CI; quantitative data are presented as LSM \pm SEM or 95\% CI, unless otherwise stated. All explanatory variables and their interactions were considered significant if $P \leq 0.05$, and $0.05<P \leq 0.10$ was considered a tendency.

\section{RESULTS}

\section{Disease Incidence and System Performance}

Of 1,121 cows enrolled in the study, $41(3.7 \%)$ were removed from the data set due to tag malfunction or misplacement during data collection. Thus, 1,080 cows were included in the final data set for analysis. Of those, $42 \%(\mathrm{n}=451)$ had no health disorders during the observation period and were included in the ND group, whereas $58 \%(\mathrm{n}=629)$ had at least 1 health disorder event during the study. Of the cows with health disorders, $70 \%$ had only one event and $30 \%$ had more than one.

Table 2 summarizes the incidence of metabolic and digestive disorders by type of disorder, DIM at CD, the sensitivity of HIS to identify cows with metabolic and digestive disorders, and the mean interval between the first positive HIS outcome (positive outcomes only) and CD. Except for indigestion, the sensitivity of HIS to detect cows with DA, ketosis, and all metabolic disorders combined was greater than $90 \%$, and cows were identified earlier $(P<0.01)$ based on HIS than through CD by farm personnel. We observed no differences in sensitivity for cows with only the metabolic and digestive disorder of interest compared with cows with more than 1 disorder during the range of interest around CD.

\section{Rumination, Activity, HIS, and Milk Production Relative to Clinical Diagnosis}

Daily rumination time, activity, and HIS patterns from -5 to $5 \mathrm{~d}$ after $\mathrm{CD}$ for each disorder of interest are shown in Figures 2 and 3. In all cases, parity and group-by-parity interaction were not significant. For cows diagnosed with DA (Figure 2A, B, C), we observed an interaction between group and day $(P<0.01)$ for rumination. Only 1 cow with DA was in the HI- group. Data from this cow are presented in figures but were not included in the statistical analysis. Rumination was lower for cows in the in HI+ group $(\mathrm{n}=40)$ than in the ND group $(\mathrm{n}=435)$ from -5 to $1 \mathrm{~d}$ relative to $\mathrm{CD}$, reaching a nadir $(\sim 216 \mathrm{~min} / \mathrm{d})$ on $\mathrm{d} 0$. Thereafter, rumination increased for cows in the HI+ group until it was similar to that of cows in the ND group from 2 to 5 $\mathrm{d}$ after $\mathrm{CD}$. We detected an interaction between group and day $(P<0.01)$ for activity. Cows in the HI+ group had lower activity than cows in the ND group from -5 to $4 \mathrm{~d}$ relative to $\mathrm{CD}$, with the lowest value $(\sim 360$ arbitrary units/d) on d 0 . We detected an interaction between group and day $(P<0.01)$ for HIS, because 


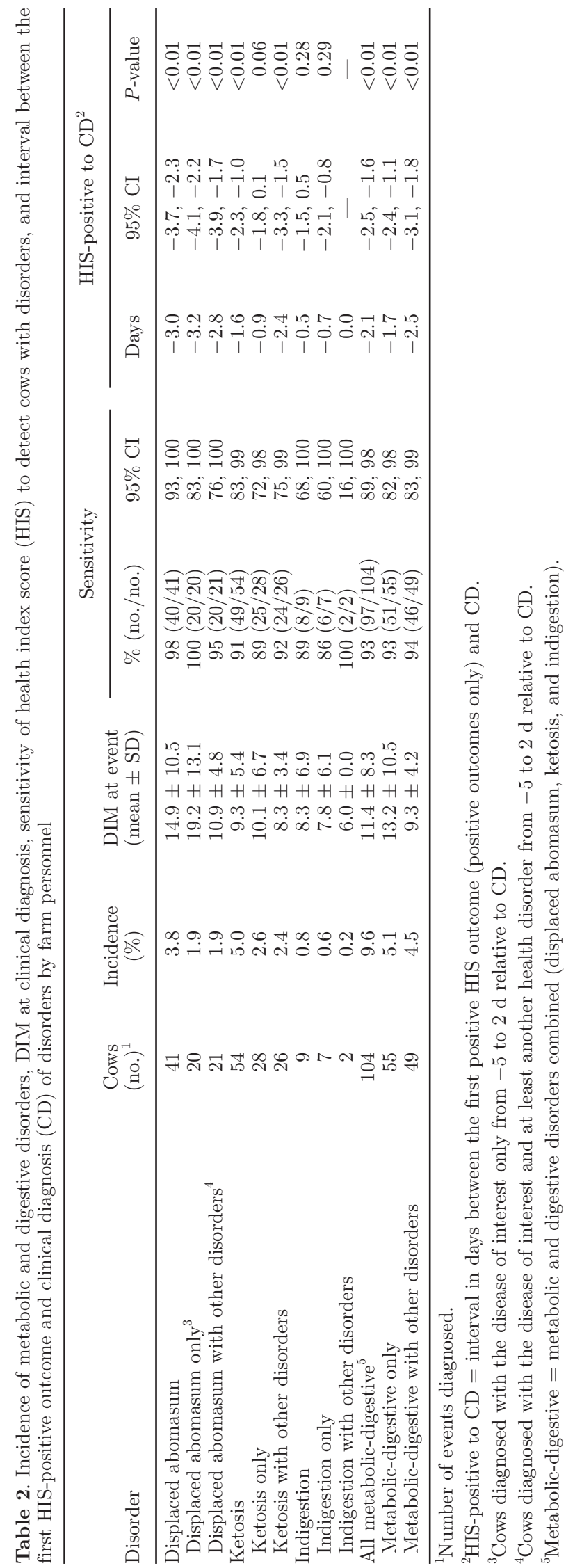

cows in the HI+ group had lower HIS than cows in the ND group from -5 to $2 \mathrm{~d}$ after CD, reaching the lowest value on $\mathrm{d} 1$ ( $\sim 75$ units). For cows with DA, HIS was also affected $(P<0.01)$ by the occurrence of ketosis. Cows with ketosis had lower HIS than cows without ketosis during the period of interest around CD (86 \pm 2 vs. $93 \pm 2$ arbitrary units, respectively).

For cows diagnosed with ketosis (Figure 2D, E, F), we observed an interaction between group and day $(P<$ $0.01)$ for rumination. Cows in the HI+ $(\mathrm{n}=44)$ group had lower rumination time than cows in the ND group $(\mathrm{n}=435)$ during the entire period analyzed, reaching a nadir $(\sim 275 \mathrm{~min} / \mathrm{d})$ on $\mathrm{d}-1$. Cows in the HI- group $(\mathrm{n}=5)$ had higher rumination time than cows in the $\mathrm{HI}+$ group from -3 to 5 d relative to $\mathrm{CD}$, but we observed no differences with the ND group. We detected an interaction between group and day $(P=0.01)$ for activity in cows with ketosis. Daily activity was lower for cows in the HI+ group than in the ND group during the entire period analyzed, and the HI- group had similar activity to cows in the HI+ and ND groups. Daily activity for cows in the HI+ group reached its lowest value on d 1 ( $\sim 380$ arbitrary units/d) relative to $\mathrm{CD}$. We observed an interaction between group and day $(P<0.01)$ for HIS. Cows with ketosis in the HI+ group had lower HIS than cows in the ND group from -3 to $5 \mathrm{~d}$ relative to $\mathrm{CD}$ and lower HIS than cows in the HI- group from -2 to 3 d relative to $\mathrm{CD}$. The nadir for cows in the HI+ group was observed on $\mathrm{d} 0$ and 1 ( 74 units of HIS). For cows with ketosis, HIS was also affected by the occurrence of DA and indigestion, because cows with DA had lower HIS $(P=0.01)$ than cows without DA during the period of interest around $\mathrm{CD}$ (78 \pm 3 vs. $85 \pm 2$ arbitrary units, respectively), and cows with indigestion had lower HIS $(P<0.01)$ than cows without indigestion during the period of interest around CD (71 \pm 4 vs. $91 \pm 1$ arbitrary units, respectively).

For cows with indigestion (Figure 3A, B, C), we observed an effect of group $(P<0.01)$ and a tendency for an interaction between group and day $(P=0.06)$ on rumination. Only 1 cow with indigestion was in the HI- group. Data from this cow are presented in figures but were not included in the statistical analysis. Cows in the HI+ group $(\mathrm{n}=8)$ had lower rumination than cows in the ND group $(\mathrm{n}=435)$, reaching a nadir $(\sim 304 \mathrm{~min} / \mathrm{d})$ on d 0 . We observed an effect of group ( $P$ $<0.01$ ) for activity, because activity was lower for cows in the HI+ than the ND group, with the lowest value ( $\sim 382$ arbitrary units/d) on d 1. For HIS we observed an interaction between group and day $(P<0.01)$. Cows in the HI+ group had lower HIS than cows in the ND group from 0 to $4 \mathrm{~d}$ after $\mathrm{CD}$, with the lowest values on d 1 and 2 ( $\sim 74$ units of HIS). For cows with indigestion, 

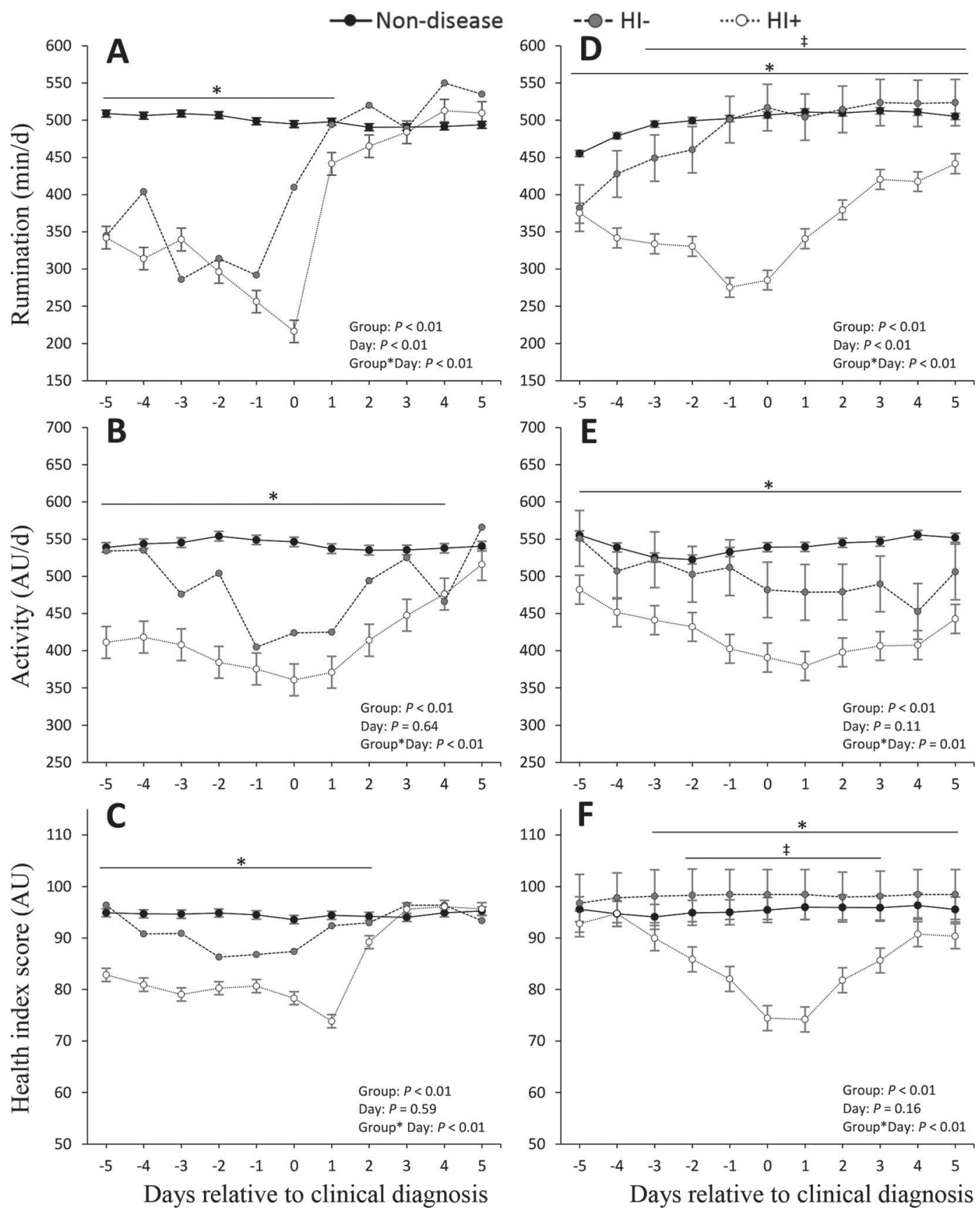

Figure 2. Daily rumination time (A), daily activity (B), and health index score (HIS; C) patterns from -5 to 5 d relative to clinical diagnosis (CD) for cows that developed a displaced abomasum (DA) and cows in the nondisease (ND) group ( $\mathrm{n}=435$; no health disorders during the study period). Cows with a DA were separated based on HIS patterns around the day of CD into HI+ $(\mathrm{n}=40)$ and HI $-(\mathrm{n}=1)$ groups. Cows were assigned to the HI+ or HI- group if they presented a HIS of $<86$ or $\geq 86$ arbitrary units (AU), respectively, during the $5 \mathrm{~d}$ before, the day of, and $2 \mathrm{~d}$ after CD. Data from the single cow in the HI- group are presented in the figure but were not included in the statistical analysis. For cows in the ND group, the average DIM at CD for cows with DA was considered "day 0." Daily rumination time (D), daily activity (E), and HIS (F) patterns from -5 to $5 \mathrm{~d}$ relative to CD for cows that developed ketosis and cows in the ND group ( $\mathrm{n}=435$ ). Cows with ketosis were assigned to the HI+ $(\mathrm{n}=44)$ and HI- group $(\mathrm{n}=5)$ following the same criteria as for cows with a DA. For cows in the ND group, the average DIM at CD for cows with ketosis was considered "day 0." Values are presented as LSM \pm SEM. Within a day, pairwise comparisons that were statistically different $(P \leq 0.05)$ based on LSD are represented as follows: *control vs. HI+; $\ddagger$ HI + vs. HI-. 


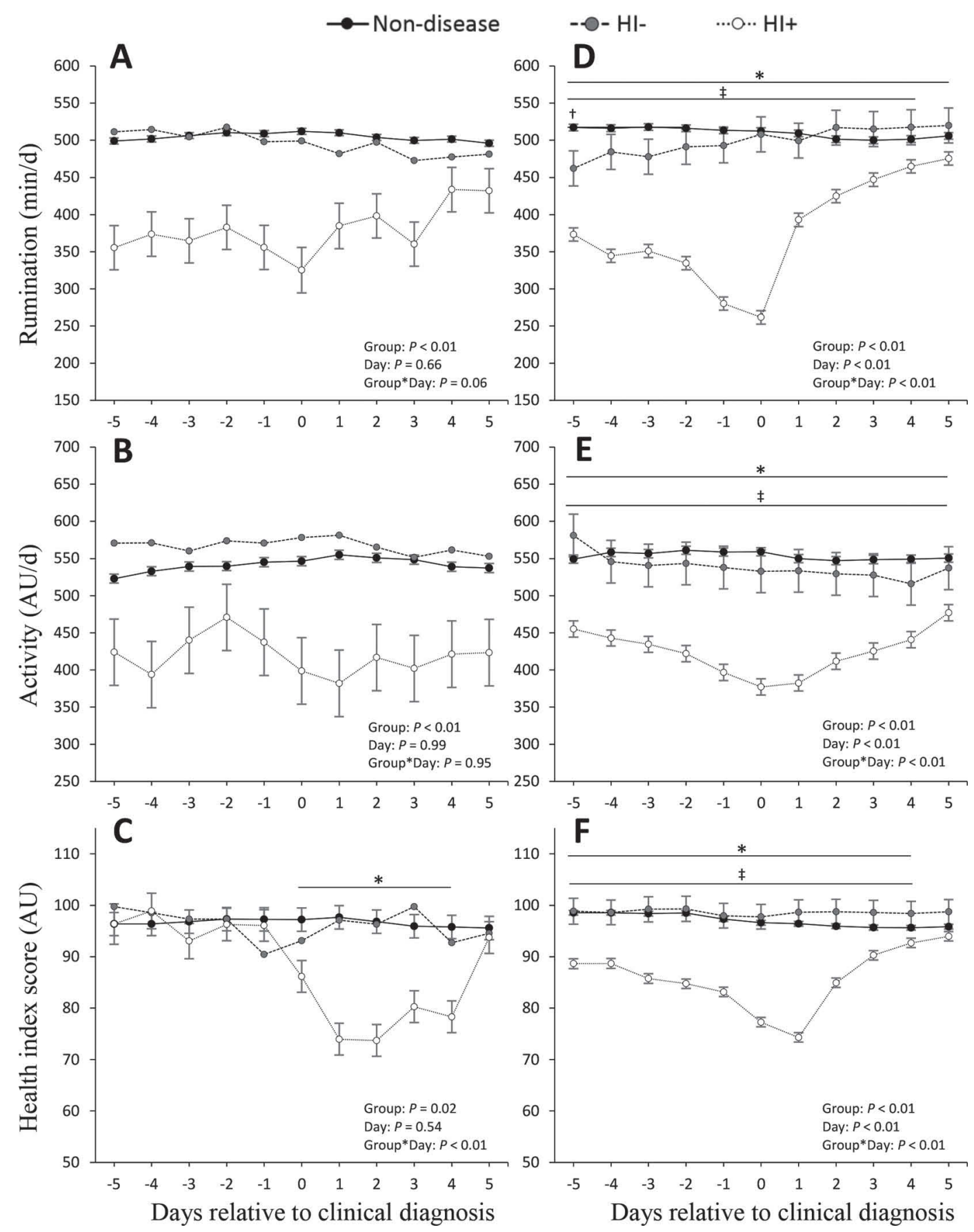

Figure 3. Daily rumination time (A), daily activity (B), and health index score (HIS; C) patterns from -5 to 5 d relative to clinical diagnosis (CD) for cows diagnosed with indigestion and cows in the nondisease (ND) group ( $\mathrm{n}=435$; no health disorders during the study period). Cows with indigestion were separated based on HIS patterns around the day of CD into HI+ $(\mathrm{n}=8)$ and HI- $(\mathrm{n}=1)$ groups. Cows were assigned to the HI+ or HI- group if they presented a HIS of $<86$ or $>86$ arbitrary units (AU), respectively, during the $5 \mathrm{~d}$ before, the day of, and $2 \mathrm{~d}$ after CD. Data from the single cow in the HI- group are presented in the figure but were not included in the statistical analysis. For cows in the ND group, the average DIM at CD for cows with indigestion was considered "day 0." Daily rumination time (D), daily activity (E), and HIS (F) patterns from -5 to $5 \mathrm{~d}$ relative to $\mathrm{CD}$ for cows that developed metabolic disorders, digestive disorders, or both, and cows in the ND group ( $\mathrm{n}=$ 435). Cows with disorders were subdivided based on their health index score pattern around the day of CD into HI+ (n = 92) and HI- groups $(\mathrm{n}=7)$ following the same criteria as for cows with indigestion. For cows in the ND group, the average DIM at CD for cows that had metabolic disorders, digestive disorders, or both was considered "day 0." Values are presented as LSM \pm SEM. Within a day, pairwise comparisons that were statistically different $(P \leq 0.05)$ based on LSD are represented as follows: * control vs. HI+; †control vs. HI-; $\ddagger$ HI+ vs. HI-. 
HIS was also affected $(P<0.01)$ by the occurrence of pneumonia. Cows with pneumonia had lower HIS than cows without pneumonia during the period of interest around CD (72 \pm 5 vs. $94 \pm 2$ arbitrary units, respectively).

For all metabolic and digestive events combined (Figures $3 \mathrm{D}, 3 \mathrm{E}$, and $3 \mathrm{~F}$ ), we observed an interaction between group and day $(P<0.01)$ for rumination. Daily rumination time was lower for cows in the HI+ group $(\mathrm{n}=92)$ than in the ND group $(\mathrm{n}=435)$ during the entire period analyzed, reaching a nadir $(\sim 262 \mathrm{~min} / \mathrm{d})$ on $\mathrm{d} 0$. Cows in the HI- group $(\mathrm{n}=7)$ had higher rumination time than cows in the HI+ group from -5 to $4 \mathrm{~d}$ relative to $\mathrm{CD}$. Compared with the ND group, cows in the HI- group had lower rumination time on $\mathrm{d}-5$ relative to $\mathrm{CD}$. Rumination time for cows with metabolic and digestive disorders was also affected by the occurrence of pneumonia, because cows with pneumonia had reduced rumination time $(P<0.01)$ than cows without pneumonia during the period of interest around CD (325 \pm 31 vs. $463 \pm 6 \mathrm{~min} / \mathrm{d}$, respectively). Activity patterns were also affected by the interaction between group and day $(P<0.01)$. Daily activity was lower for cows in the HI+ group than for cows the HIand ND groups during the entire period analyzed, with the lowest value $(\sim 377$ arbitrary units/d) on d 0 relative to CD. Finally, we observed an interaction between group and day $(P<0.01)$ for HIS. Cows in the HI+ group had lower HIS than cows in the HI- and the ND groups from -5 to $4 \mathrm{~d}$, after CD reaching a nadir ( $\sim 74$ units of HIS) on d 1. For cows with metabolic and digestive disorders, HIS was also affected by the occurrence of metritis, pneumonia, and lameness during the period of interest around CD. Cows with metritis had lower HIS $(P=0.05)$ than cows without metritis ( $80 \pm 2$ vs. $83 \pm 3$ arbitrary units, respectively). Cows with pneumonia had lower HIS $(P<0.01)$ than cows without pneumonia ( $73 \pm 3$ vs. $91 \pm 2$ arbitrary units, respectively). Cows with lameness had lower HIS $(P<$ $0.01)$ than cows without lameness $(77 \pm 4$ vs. $86 \pm 1$ arbitrary units, respectively).

Table 3 includes differences (in units of measurement) and percent change between $5 \mathrm{~d}$ before $\mathrm{CD}$ and the day of the nadir for rumination, activity, HIS, and milk production for all metabolic and digestive disorders combined.

\section{Plasma Concentrations of NEFA, BHB, Calcium, and Haptoglobin}

Plasma concentration of NEFA, BHB, Ca, and haptoglobin relative to the day of calving for cows that developed DA are presented in Figure 4. Only 1 cow had a DA in the HI- group. Data from this cow are

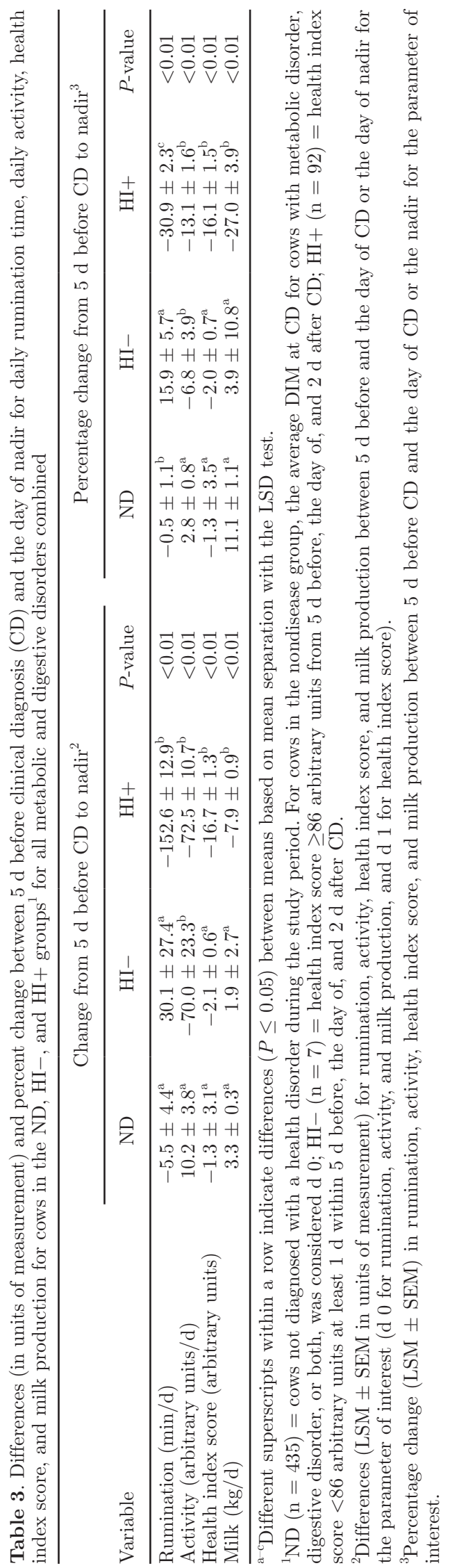



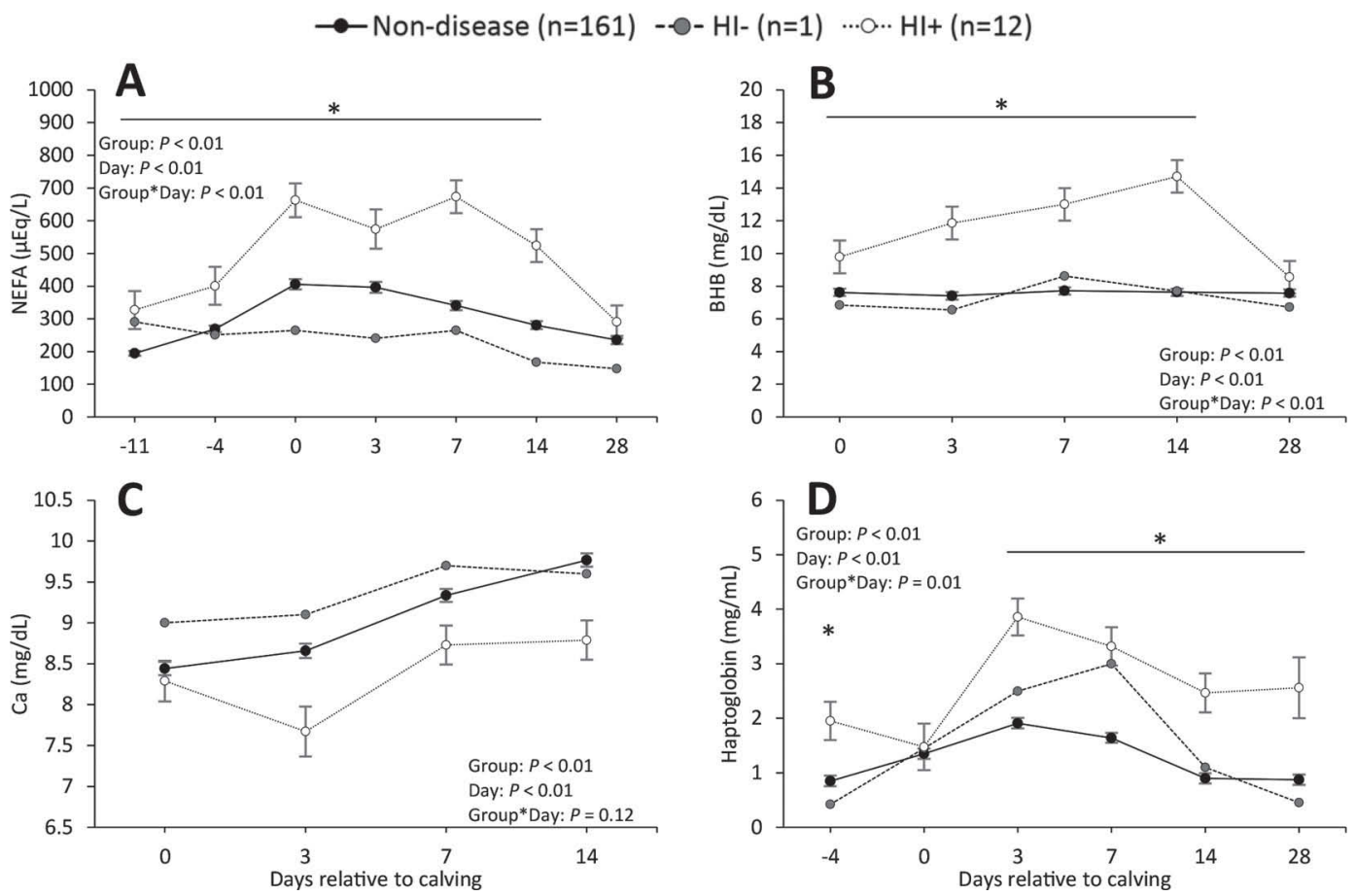

Figure 4. Plasma concentrations of nonesterified fatty acids (NEFA; A), BHB (B), total Ca (C), and haptoglobin (D) for cows that developed a displaced abomasum $(\mathrm{DA})$ and cows in the ND group $(\mathrm{n}=161$; no health disorders during the study period). Cows with a DA were separated based on health index score (HIS) patterns around the day of clinical diagnosis (CD) into HI+ $(\mathrm{n}=12)$ and HI- (n = 1) groups. Cows were assigned to the HI+ or HI- group if they presented a HIS of $<86$ or $\geq 86$ arbitrary units, respectively, during the $5 \mathrm{~d}$ before, the day of, and 2 $\mathrm{d}$ after CD. Data from the single cow in the HI- group are presented in the figure but were not included in the statistical analysis. Values are presented as LSM \pm SEM. Within a day, differences $(P \leq 0.05)$ for pairwise comparisons between the ND and HI+ groups are represented by an asterisk $(*)$.

presented in figures but were not included in the statistical analysis. For NEFA, we observed an interaction between group and day $(P<0.01)$. Plasma concentrations of NEFA were greater for cows in the HI+ group than in the ND group from -11 to $14 \mathrm{~d}$ after calving. Concentrations of NEFA for cows in the HI+ group peaked $0 \mathrm{~d}(\sim 663 \mu \mathrm{Eq} / \mathrm{L})$ and $7 \mathrm{~d}$ after calving $(\sim 673$ $\mu \mathrm{Eq} / \mathrm{L})$, with differences of $\sim 257 \mu \mathrm{Eq} / \mathrm{L}$ and $\sim 332$ $\mu \mathrm{Eq} / \mathrm{L}$ between the HI+ and ND groups 0 and $7 \mathrm{~d}$ after calving, respectively. In addition, NEFA concentrations were also affected $(P<0.01)$ by the occurrence of ketosis. Cows with ketosis had greater NEFA concentrations than cows without ketosis ( $675 \pm 62$ vs. $308 \pm 5 \mu \mathrm{Eq} / \mathrm{L}$, respectively). For concentrations of BHB in plasma, we also observed an interaction between group and day $(P<0.01)$. Plasma BHB concentrations were greater for cows in the HI+ group than in the ND group from calving to $14 \mathrm{~d}$ after calving. Concentrations of $\mathrm{BHB}$ reached their highest value $(\sim 15 \mathrm{mg} / \mathrm{dL}) 14 \mathrm{~d}$ after calving. The greatest difference in BHB concentrations between the $\mathrm{HI}+$ and ND groups was observed at $14 \mathrm{~d}$ after calving $(7 \mathrm{mg} / \mathrm{dL})$. For concentrations of total Ca in plasma, we observed an effect of group $(P<0.01)$, but no group-by-day interaction $(P=0.12)$. Cows in the HI+ group had lower total Ca concentrations than cows in the ND group during the entire sampling period, reaching nadir concentrations $(\sim 7.7 \mathrm{mg} / \mathrm{dL})$ and the greatest difference compared to cows in the ND group $(\sim 1.0 \mathrm{mg} / \mathrm{dL}) 3 \mathrm{~d}$ after calving. Concentrations of haptoglobin in plasma were affected by the interaction between group and day $(P=0.01)$. Cows in the $\mathrm{HI}+$ group had greater haptoglobin concentrations than cows in the ND group $4 \mathrm{~d}$ before calving and from 3 to $28 \mathrm{~d}$ after calving, reaching peak concentrations $(\sim 3.9$ $\mathrm{mg} / \mathrm{mL}$ ) and the greatest difference compared to cows in the ND group $(\sim 2.0 \mathrm{mg} / \mathrm{mL})$ at $3 \mathrm{~d}$ after calving.

Plasma concentration of NEFA, BHB, Ca, and haptoglobin relative to the day of calving for cows that developed ketosis are presented in Figure 5. No cows with ketosis and from the HI- group were sampled (or represented). For plasma NEFA concentrations, we observed an interaction between group and day $(P$ $<0.01)$. Cows in the HI+ group had greater NEFA concentrations than cows in the ND group during the entire period analyzed. Peak concentrations for NEFA were observed $0 \mathrm{~d}(\sim 907 \mu \mathrm{Eq} / \mathrm{L})$ and $7 \mathrm{~d}(\sim 876 \mu \mathrm{Eq} / \mathrm{L})$ after calving, with differences between the $\mathrm{HI}+$ and $\mathrm{ND}$ groups of $\sim 509 \mu \mathrm{Eq} / \mathrm{L}$ and $\sim 543 \mu \mathrm{Eq} / \mathrm{L} 0$ and $7 \mathrm{~d}$ after 
calving, respectively. In addition, NEFA concentrations were affected by the occurrence of metritis and mastitis, because cows with metritis had greater $(P=0.03)$ NEFA concentrations than cows without metritis (287 \pm 45 vs. $144 \pm 79 \mu \mathrm{Eq} / \mathrm{L}$, respectively), and cows with mastitis had greater $(P<0.01)$ NEFA concentrations than cows without mastitis $(407 \pm 29$ vs. $124 \pm 99$ $\mu \mathrm{Eq} / \mathrm{L}$, respectively). For plasma BHB concentrations, we observed an interaction between group and day $(P$ $<0.01)$. Cows in the HI+ group exhibited greater BHB concentrations than cows in the ND group from 0 to $28 \mathrm{~d}$ after calving, with concentrations of $\sim 16 \mathrm{mg} / \mathrm{dL}$ at $14 \mathrm{~d}$ after calving. The greatest differences for BHB concentrations between the $\mathrm{HI}+$ and ND groups were observed at $14 \mathrm{~d}(8.3 \mathrm{mg} / \mathrm{dL})$ after calving. In addition, BHB concentrations were affected by the occurrence of metritis, mastitis, and indigestion. Cows with metritis had greater $(P=0.04)$ BHB concentrations than cows without metritis $(10.5 \pm 1.1$ vs. $8.2 \pm 1.7 \mathrm{mg} / \mathrm{dL}$, respectively), cows with mastitis had greater $(P<0.01)$ BHB concentrations than cows without mastitis (12.1 \pm 1.0 vs. $6.6 \pm 2.0 \mathrm{mg} / \mathrm{dL}$, respectively), and cows with indigestion had greater $(P<0.01)$ BHB concentrations than cows without indigestion $(12.4 \pm 2.0$ vs. $6.3 \pm$ $1.0 \mathrm{mg} / \mathrm{dL}$, respectively). For plasma total Ca concentration, we observed a group-by-day interaction $(P=$
0.01). Total Ca concentrations were lower for cows in the HI+ group than cows in the ND group from 7 to 14 $\mathrm{d}$ after calving, with the greatest differences between the $\mathrm{HI}+$ and ND groups 7 and $14 \mathrm{~d}(\sim 0.6 \mathrm{mg} / \mathrm{dL})$ after calving. Total Ca concentrations were also affected by the occurrence of indigestion, because cows with indigestion had lower $(P<0.01)$ Ca concentrations than cows without indigestion $(7.8 \pm 0.4 \mathrm{mg} / \mathrm{dL}$ vs. $8.9 \pm$ 0.1 , respectively) during the period of interest around $\mathrm{CD}$ of ketosis. For plasma haptoglobin concentrations, we observed an interaction between group and day $(P$ $<0.01)$. Cows in the HI+ group had greater haptoglobin concentrations than cows in the ND group from 3 to $14 \mathrm{~d}$ after calving, with the highest concentration $(\sim 3.6 \mathrm{mg} / \mathrm{mL}) 3 \mathrm{~d}$ after calving. Haptoglobin concentrations were also affected by the occurrence of DA and pneumonia. Cows with a DA had greater $(P<0.01)$ haptoglobin concentrations than cows without DA (3.9 \pm 0.6 vs. $2.4 \pm 0.4 \mathrm{mg} / \mathrm{mL}$, respectively), and cows with pneumonia had greater $(P<0.01)$ haptoglobin concentrations than cows without pneumonia (4.1 \pm 0.8 vs. $2.3 \pm 0.2 \mathrm{mg} / \mathrm{mL}$, respectively).

Data for cows with indigestion are not presented, because they were available from only 3 cows in the $\mathrm{HI}+$ group and 1 cow in the HI- group. Briefly, cows in the HI+ group had greater NEFA concentrations than
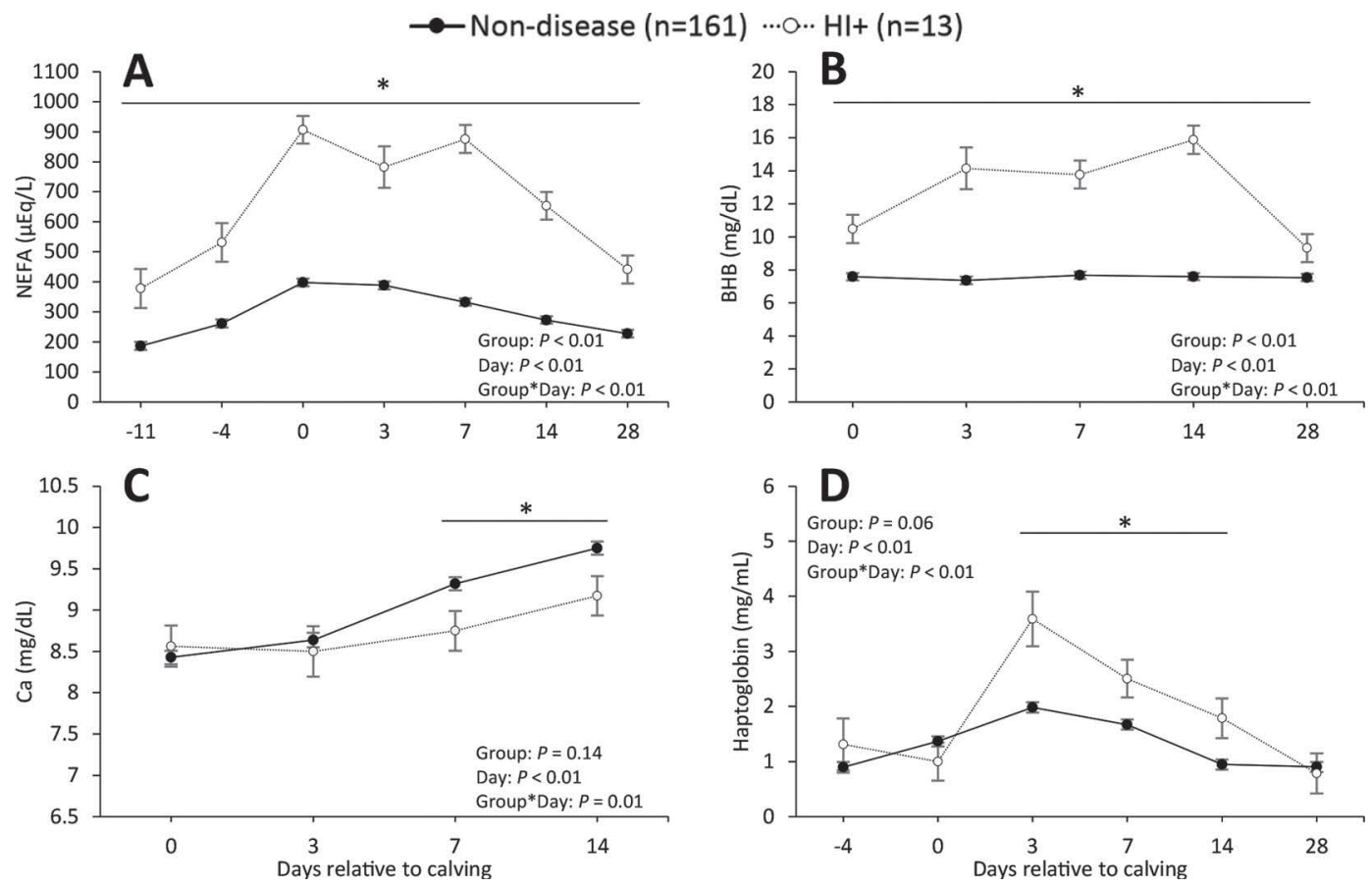

Figure 5. Plasma concentrations of nonesterified fatty acids (NEFA; A), BHB (B), total Ca (C), and haptoglobin (D) for cows that developed a case of ketosis $(n=13)$ and cows in the ND group $(n=161$; no health disorders during the study period). All cows with ketosis were from the $\mathrm{HI}+$ group. Cows were assigned to the HI+ or HI- group if they had a health index score of $<86$ or $\geq 86$ arbitrary units, respectively, during the $5 \mathrm{~d}$ before, the day of, and $2 \mathrm{~d}$ after clinical diagnosis. Values are presented as LSM \pm SEM. Within a day, pairwise differences $(P \leq 0.05)$ between the ND and $\mathrm{HI}+$ groups are represented by an asterisk $(*)$. 
cows in the ND group from 0 to 14 DIM $(P<0.01)$, greater BHB from 3 to 14 DIM $(P<0.01)$, lower total Ca concentrations $(P<0.01)$, and no differences in haptoglobin concentrations $(P=0.20)$.

\section{DISCUSSION}

The timely detection of cows with health disorders during the early postpartum period requires consistent implementation of a comprehensive health-monitoring program. This observational cohort study was designed to evaluate the feasibility of using an automated rumination and physical activity monitoring system to identify lactating dairy cows with metabolic and digestive disorders. Using CD by farm personnel following specific criteria for defining health disorders and standard operating procedures as reference, we found the sensitivity of the system based on HIS to be high-ranging, from $89 \%$ to $98 \%$ for each individual disorder and $93 \%$ for all disorders combined. We observed the greatest sensitivity for cows that developed DA, intermediate sensitivity for cows with ketosis, and lowest sensitivity for cows with indigestion. Likely, the differences in sensitivities are a reflection of the severity of the disorder or the number of cows included in the study for some of the specific conditions. For example, an episode of DA is more disruptive to cow health than an episode of ketosis. In addition, it is possible that cows with a DA were also ketotic before the DA was diagnosed (Geishauser et al., 1997; LeBlanc et al., 2005) affecting all of the parameters evaluated for several days before CD. For indigestion, we speculate that the low number of cows with the disorder may have been the main contributor to the lower sensitivity, because only 1 cow out of 9 was not flagged based on HIS. Interestingly, we did not observe higher sensitivity of HIS for cows with more than 1 health disorder, in spite of the fact that the occurrence of more than 1 disorder at the same time caused changes to patterns of rumination, activity, and HIS. These observations provided additional evidence that the sole occurrence of the metabolic and digestive disorders evaluated caused alterations to rumination and activity of sufficient magnitude to trigger a HIS alert.

We also speculate that cows not flagged based on HIS had a less severe episode of the disorder. Although we did not establish a severity score, this notion is based on the daily pattern of the parameters evaluated during the $5 \mathrm{~d}$ before and $5 \mathrm{~d}$ after $\mathrm{CD}$ for the ND group and those with the disorders. For cows with ketosis and all cows with metabolic and digestive disorders combined, cows in the HI- group demonstrated patterns of rumination, activity, and HIS that were not different from cows in the ND group for most of the days evaluated. More importantly, the rumination and activity patterns used to calculate HIS did not show a dramatic reduction in the days before CD. This was particularly relevant to the AHMS used in this study, because it did not compare cows to their herd mates; instead, it used changes in pattern of rumination and activity for individual cows. For the single cow that had a DA and was not flagged by the AHMS, rumination and activity showed a marked decline, resulting in a reduction in HIS. However, this cow was not flagged because the HIS was not below the 86-point cutoff on any day evaluated. Interestingly, the nadir for rumination, activity, and HIS for this cow was observed $1 \mathrm{~d}$ before $\mathrm{CD}$, and rumination showed a dramatic rebound before surgical treatment.

The notion that the severity of the disorders was different for cows in the $\mathrm{HI}-$ and $\mathrm{HI}+$ groups is also supported by the changes in rumination, activity, HIS, and milk production between $5 \mathrm{~d}$ before $\mathrm{CD}$ and the day of the nadir for each parameter. Except for activity, which was similar for cows in the HI- and HI+ groups, we observed minor reductions or slight increments in rumination, HIS, and milk production for cows in the HI- and ND groups. This is in sharp contrast to the dramatic reductions in rumination, activity, and HIS for cows in the HI+ group. Notably, milk production in the HI+ group decreased by $8 \mathrm{~kg}$ or $27 \%$ from $5 \mathrm{~d}$ before to the day of $\mathrm{CD}$. Although these results may question the validity of the $\mathrm{CD}$ and the economic benefits of treating cows not flagged by the AHMS, there may have been potential benefits to cow health and welfare that should not be overlooked if the cows had truly developed the disorder. Our study does not provide evidence that cows in the HI- group did not have a reduction in milk production or overall performance beyond the days included in the analysis. In this regard, previous studies have documented reductions in milk production in cows with ketosis and DA earlier than 5 d from CD (Lucey et al., 1986; Rajala-Schultz et al., 1999; Edwards and Tozer, 2004).

Another major objective of our study was to determine the interval between the time of the first alert based on HIS and the day of CD. Cows in the HI+ group for each disorder showed differences in rumination and activity compared with cows in the ND group as early as $5 \mathrm{~d}$ before $\mathrm{CD}$, suggesting that some cows began experiencing consequences of the disorder more than 5 $\mathrm{d}$ before CD. Whether this is the result of very early manifestation of the disorder or because these cows did not recover after calving is unknown. Remarkably, cows with DA and ketosis were flagged based on HIS before $\mathrm{CD}$, whereas cows with indigestion were detected by HIS at a similar time to CD. Previous studies have also reported dramatic reductions in rumination (Gaspardy 
et al., 2014) and activity (Edwards and Tozer, 2004) 5 to $6 \mathrm{~d}$ before the occurrence of ketosis and DA in dairy cows. For activity, however, we observed a more consistent decline until the day of CD than previously reported (Edwards and Tozer, 2004). These differences could be explained, at least in part, by differences between studies for the timing and frequency of pen moves around the day of CD and differences between the activity recording systems. Unlike cows with DA and ketosis, the pattern of decline for HIS for cows with indigestion was not as substantial until the day of $\mathrm{CD}$, when a sudden drop in mean HIS below 86 points was observed. This sudden, rather sustained, decline in HIS before CD was the most likely reason for the short time frame between identification by the AHMS and CD.

Earlier identification of cows with health disorders presents opportunities and challenges. Detecting a health disorder at an early stage and before the manifestation of clear clinical signs may benefit cows by improving overall treatment response and reducing the negative long-term consequences of disease on overall cow health and performance (Fourichon et al., 1999; Seifi et al., 2011). Nevertheless, detecting cows with a health disorder at its very early stages may create new challenges, because farm personnel must determine whether the cow truly has a disorder and what the disorder is in the absence of clear clinical signs. Under these circumstances, the selection of a treatment strategy may be limited or less specific than when clinical signs are evident. Additional tests to confirm the presence of subclinical disorders or underlying predisposing factors for clinical diseases may facilitate decision-making (LeBlanc et al., 2005; McArt et al., 2011, 2012). Future research is warranted to establish criteria for differentiating and treating specific health disorders based on the information provided by the AHMS.

In our study, the pattern of rumination, activity, and HIS for each disorder of interest was useful not only to explore potential differences among cows that were or were not flagged based on HIS, but also to document the pattern for these parameters around the timing of CD. Interestingly, cows in the HI+ group showed the nadir for the parameters measured on the day of or the day after $\mathrm{CD}$, except rumination for ketosis observed on $\mathrm{d}-1$. In agreement, a recent study reported that cows with subclinical ketosis (case definition not reported) had reduced daily rumination time before and during an episode of the disease (accumulated over 6 d) followed by a substantial rebound of $\sim 60$ to $100 \mathrm{~min}$ per d after resolution of the disorder (Gaspardy et al., 2014). Liboreiro et al. (2015) also reported differences between cows without a health disorder and cows with subclinical ketosis (BHB in blood $>1,000 \mu \mathrm{mol} / \mathrm{L}$ ) at 8 and 11 DIM, which was within the range of DIM for cases of ketosis in our study and others (McArt et al., 2012).

For all parameters of interest, their respective nadir was followed by a rebound of different magnitude depending on the disorder and parameter beginning on the day of or the first $2 \mathrm{~d}$ after treatment. Likely, this is the reflection of an immediate response to treatment. At the moment, however, it is unknown if cows would have had a further reduction in the parameters monitored if left untreated, because every cow received some form of treatment. Interestingly, Gonzalez et al. (2008) observed a more prolonged nadir and slower feed intake recovery after CD for a non-treated than a treated cow with ketosis.

In spite of the substantial rebound and recovery to levels observed $5 \mathrm{~d}$ before $\mathrm{CD}$ for cows with ketosis, the mean for all parameters evaluated did not fully match that of cows in the ND group $5 \mathrm{~d}$ after treatment. This could be a reflection of incomplete recovery or the fact that some cows developed 2 disorders sequentially. Indeed, $43 \%$ of the cows diagnosed with ketosis in our study were also diagnosed with DA within $5.1 \mathrm{~d}$ after the diagnosis of ketosis. This is not surprising, because ketosis is a major risk factor for DA in lactating dairy cows (LeBlanc et al., 2005; Seifi et al., 2011). On the other hand, the pattern of rumination and activity after CD and treatment may be useful for evaluating the course of disease and response to treatment. A better understanding of the specific patterns of rumination, activity, or parameters created based on their combination (e.g., HIS) for cows that develop different health disorders in the early postpartum period may help in designing specific algorithms to predict the type of disorder the cow had before manifestation of clinical signs.

The association between markers of energy status (NEFA and BHB), mineral balance (Ca), and systemic inflammation (haptoglobin) with the occurrence of metabolic and digestive disorders in dairy cows has been well established (LeBlanc et al., 2005; Huzzey et al., 2011; Seifi et al., 2011; Chapinal et al., 2012). Therefore, the differences in circulating concentrations of NEFA, BHB, Ca, and haptoglobin observed for cows in the ND group compared to cows that developed metabolic and digestive disorders was expected. Because it was not possible to predict which cows would develop health disorders and when, blood samples were collected from a convenient sample of cows on specific days before and after calving. As a result, it was not possible to report the levels of the markers of interest around the timing of occurrence of each particular disorder. However, based on DIM at CD, the pattern of circulating concentrations of all markers was in agreement with the early and dramatic changes observed for the parameters monitored by the AHMS. Within 0 to 3 until 14 
DIM, NEFA, BHB, Ca, and haptoglobin concentrations for cows in the HI+ group were different than in the ND group, supporting the notion that these cows were in a more severe plane of negative energy balance, were Ca deficient, and were undergoing more severe systemic inflammation than cows without a health disorder. For cows in the HI- group, the limited number of observations precluded drawing any relevant conclusions, but the notion that these cows were less severely affected by the observed health disorder was partially supported. Whether rumination and activity were lower because of the underlying condition affecting the cow and its effect on feeding behavior, or because of the altered levels of the markers measured in our study, is unknown. Similar associations between blood markers of metabolic and health status and rumination and activity have been reported for lactating dairy cows (Soriani et al., 2012; Liboreiro et al., 2015) but no cause-and-effect relationships were established. When all cows with metabolic and digestive disorders identified based on HIS were combined, we observed differences compared to cows in the ND group for NEFA and haptoglobin before calving. These observations were not surprising, because elevated levels of NEFA and haptoglobin in the immediate prepartum period have been identified as risk factors for DA and ketosis (LeBlanc et al., 2005; Ospina et al., 2010; Huzzey et al., 2011).

\section{CONCLUSIONS}

Our findings suggest that monitoring rumination time and physical activity could be useful for identifying cows with metabolic and digestive disorders in the early postpartum period. The HIS created based on rumination and activity by the AHMS showed high sensitivity for identifying cows with metabolic and digestive disorders, and it identified cows with ketosis and DA earlier than CD by farm personnel. The patterns of rumination, activity, and HIS from -5 to $5 \mathrm{~d}$ after $\mathrm{CD}$ of the disorder of interest were characterized by marked differences compared to cows in the ND group as early as $5 \mathrm{~d}$ before $\mathrm{CD}$ and showed a nadir around the timing of CD ( -1 to $1 \mathrm{~d}$ after $\mathrm{CD})$. We observed a considerable recovery for rumination, activity, and HIS after CD and treatment. For cows with clinical disorders not identified based on HIS, their rumination, activity, and milk production patterns were very similar to that of cows in the ND group and different from cows diagnosed with the disorder but flagged based on HIS. We conclude that automated health-monitoring systems that use rumination and activity are promising tools for the identification of cows with metabolic and digestive disorders in dairy farms.

\section{ACKNOWLEDGMENTS}

The authors extend their gratitude to the owners, managers, and personnel of the commercial dairy farm that participated in the study for providing access to their cows and facilities, and their collaboration with our research team. We also extend our gratitude to SCR Dairy (Madison, WI) for partial financial and logistical support to conduct this research and to Thomas Overton (Cornell University, Ithaca, NY) for providing useful feedback on the manuscript. Matias Stangaferro received partial support from a Fulbright fellowship and from Universidad Nacional del Litoral (Esperanza, Argentina).

\section{REFERENCES}

Bareille, N., F. Beaudeau, S. Billon, A. Robert, and P. Faverdin. 2003. Effects of health disorders on feed intake and milk production in dairy cows. Livest. Prod. Sci. 83:53-62.

Barkema, H. W., M. A. von Keyserlingk, J. P. Kastelic, T. J. Lam, C. Luby, J. P. Roy, S. J. LeBlanc, G. P. Keefe, and D. F. Kelton. 2015. Invited review: Changes in the dairy industry affecting dairy cattle health and welfare. J. Dairy Sci. 98:7426-7445.

Bartlett, P. C., M. Kopcha, P. Coe, N. Ames, P. Ruegg, and R. Erskine. 1995. Economic comparison of the pyloro-omentopexy vs the roll-and-toggle procedure for treatment of left displacement of the abomasum in dairy cattle. J. Am. Vet. Med. Assoc. 206:1156-1162.

Bicalho, M. L., F. S. Lima, E. K. Ganda, C. Foditsch, E. B. Meira Jr., V. S. Machado, A. G. Teixeira, G. Oikonomou, R. O. Gilbert, and R. C. Bicalho. 2014. Effect of trace mineral supplementation on selected minerals, energy metabolites, oxidative stress, and immune parameters and its association with uterine diseases in dairy cattle. J. Dairy Sci. 97:4281-4295.

Chapinal, N., S. J. Leblanc, M. E. Carson, K. E. Leslie, S. Godden, M. Capel, J. E. Santos, M. W. Overton, and T. F. Duffield. 2012. Herd-level association of serum metabolites in the transition period with disease, milk production, and early lactation reproductive performance. J. Dairy Sci. 95:5676-5682.

Edwards, J. L., and P. R. Tozer. 2004. Using activity and milk yield as predictors of fresh cow disorders. J. Dairy Sci. 87:524-531.

Espadamala, A., P. Pallares, A. Lago, and N. Silva-del-Rio. 2015. Fresh cow evaluations and treatments on California dairies. J. Dairy Sci. 98(E-Suppl. 1):330. (Abstr.)

Ferrero, F., M. Valledor, and J. Campo. 2014. Screening method for early detection of mastitis in cows. Measurement 47:855-860.

Fourichon, C., H. Seegers, N. Bareille, and F. Beaudeau. 1999. Effects of disease on milk production in the dairy cow: A review. Prev. Vet. Med. 41:1-35.

Gáspárdy, A., G. Efrat, A. C. Bajcsy, and S. G. Fekete. 2014. Electronic monitoring of rumination activity as an indicator of health status and production traits in high-yielding dairy cows. Acta Vet. Hung. 62:452-462.

Geishauser, T., K. Leslie, T. Duffield, and V. Edge. 1997. Evaluation of aspartate transaminase activity and beta-hydroxybutyrate concentration in blood as tests for prediction of left displaced abomasum in dairy cows. Am. J. Vet. Res. 58:1216-1220.

González, L. A., B. J. Tolkamp, M. P. Coffey, A. Ferret, and I. Kyriazakis. 2008. Changes in feeding behavior as possible indicators for the automatic monitoring of health disorders in dairy cows. J. Dairy Sci. 91:1017-1028.

Gröhn, Y. T., S. W. Eicker, V. Ducrocq, and J. A. Hertl. 1998. Effect of diseases on the culling of Holstein dairy cows in New York State. J. Dairy Sci. 81:966-978.

Huzzey, J. M., D. V. Nydam, R. J. Grant, and T. R. Overton. 2011. Associations of prepartum plasma cortisol, haptoglobin, fecal cor- 
tisol metabolites, and nonesterified fatty acids with postpartum health status in Holstein dairy cows. J. Dairy Sci. 94:5878-5889.

Ingvartsen, K. L. 2006. Feeding-and management-related diseases in the transition cow: Physiological adaptations around calving and strategies to reduce feeding-related diseases. Anim. Feed Sci. Technol. 126:175-213.

Kaneene, J. B., and H. S. Hurd. 1990. The national animal health monitoring system in Michigan. III. Cost estimates of selected dairy cattle diseases. Prev. Vet. Med. 8:127-140.

LeBlanc, S. 2010. Monitoring metabolic health of dairy cattle in the transition period. J. Reprod. Dev. 56:S29-35.

LeBlanc, S. J., K. E. Leslie, and T. F. Duffield. 2005. Metabolic predictors of displaced abomasum in dairy cattle. J. Dairy Sci $88: 159-170$.

Liboreiro, D. N., K. S. Machado, P. R. Silva, M. M. Maturana, T. K. Nishimura, A. P. Brandao, M. I. Endres, and R. C. Chebel. 2015. Characterization of peripartum rumination and activity of cows diagnosed with metabolic and uterine diseases. J. Dairy Sci. 98:6812-6827.

Lucey, S., G. J. Rowlands, and A. M. Russell. 1986. Short-term associations between disease and milk yield of dairy cows. J. Dairy Res. 53:7-15.

Lukas, J. M., J. Reneau, R. Wallace, and A. De Vries. 2015. A study of methods for evaluating the success of the transition period in early-lactation dairy cows. J. Dairy Sci. 98:250-262.

McArt, J. A., D. V. Nydam, and G. R. Oetzel. 2012. Epidemiology of subclinical ketosis in early lactation dairy cattle. J. Dairy Sci. 95:5056-5066.

McArt, J. A., D. V. Nydam, P. A. Ospina, and G. R. Oetzel. 2011. A field trial on the effect of propylene glycol on milk yield and resolution of ketosis in fresh cows diagnosed with subclinical ketosis. J. Dairy Sci. 94:6011-6020.

McArt, J. A., D. V. Nydam, and M. W. Overton. 2015. Hyperketonemia in early lactation dairy cattle: A deterministic estimate of component and total cost per case. J. Dairy Sci. 98:2043-2054.

Ospina, P. A., D. V. Nydam, T. Stokol, and T. R. Overton. 2010 Evaluation of nonesterified fatty acids and beta-hydroxybutyrate in transition dairy cattle in the northeastern United States: Critical thresholds for prediction of clinical diseases. J. Dairy Sci. 93:546-554.
Pinedo, P. J., A. De Vries, and D. W. Webb. 2010. Dynamics of culling risk with disposal codes reported by Dairy Herd Improvement dairy herds. J. Dairy Sci. 93:2250-2261.

Raizman, E. A., and J. Santos. 2002. The effect of left displacement of abomasum corrected by toggle-pin suture on lactation, reproduction, and health of Holstein dairy cows. J. Dairy Sci. 85:1157-1164.

Rajala-Schultz, P. J., Y. T. Gröhn, and C. E. McCulloch. 1999. Effects of milk fever, ketosis, and lameness on milk yield in dairy cows. J. Dairy Sci. 82:288-294.

Ribeiro, E. S., F. Lima, L. Greco, R. Bisinotto, A. Monteiro, M. Favoreto, H. Ayres, R. Marsola, N. Martinez, and W. Thatcher. 2013. Prevalence of periparturient diseases and effects on fertility of seasonally calving grazing dairy cows supplemented with concentrates. J. Dairy Sci. 96:5682-5697.

Risco, C., and P. Melendez Retamal. 2011. Dairy Production Medicine. Wiley-Blackwell, Oxford, UK.

Rutten, C. J., A. Velthuis, W. Steeneveld, and H. Hogeveen. 2013. Invited review: Sensors to support health management on dairy farms. J. Dairy Sci. 96:1928-1952.

Seifi, H. A., S. J. Leblanc, K. E. Leslie, and T. F. Duffield. 2011. Metabolic predictors of post-partum disease and culling risk in dairy cattle. Vet. J. 188:216-220.

Soriani, N., E. Trevisi, and L. Calamari. 2012. Relationships between rumination time, metabolic conditions, and health status in dairy cows during the transition period. J. Anim. Sci. 90:4544-4554.

Stangaferro, M. L., R. Wijma, L. S. Caixeta, M. A. Al-Abri, and J. O. Giordano. 2016a. Use of rumination and activity monitoring for the identification of dairy cows with health disorders. Part II. Mastitis. J. Dairy Sci. 99:7411-7421. http://dx.doi.org/10.3168/ jds.2016-10908.

Stangaferro, M. L., R. Wijma, L. S. Caixeta, M. A. Al-Abri, and J. O. Giordano. 2016b. Use of rumination and activity monitoring for the identification of dairy cows with health disorders. Part III. Metritis. J. Dairy Sci. 99:7422-7433. http://dx.doi.org/10.3168/ jds.2016-11352.

Vercouteren, M. M., J. H. Bittar, P. J. Pinedo, C. A. Risco, J. E. Santos, A. Vieira-Neto, and K. N. Galvao. 2015. Factors associated with early cyclicity in postpartum dairy cows. J. Dairy Sci $98: 229-239$. 